\title{
Population differentiated copy number variation of Bos taurus, Bos indicus and their African hybrids
}

Jisung Jang ${ }^{1}$, Endashaw Terefe ${ }^{2,3,4}$, Kwondo Kim5, Young Ho Lee ${ }^{1}$, Gurja Belay², Abdulfatai Tijjani3 ${ }^{3,6}$, Jian-Lin Han,8, Olivier Hanotte ${ }^{3,6,9}$ and Heebal Kim ${ }^{1,5,10^{*}}$

\begin{abstract}
Background: CNV comprises a large proportion in cattle genome and is associated with various traits. However, there were few population-scale comparison studies on cattle CNV.

Results: Here, autosome-wide CNVs were called by read depth of NGS alignment result and copy number variation regions (CNVRs) defined from 102 Eurasian taurine (EAT) of 14 breeds, 28 Asian indicine (ASI) of 6 breeds, 22 African taurine (AFT) of 2 breeds, and 184 African humped cattle (AFH) of 17 breeds. The copy number of every CNVRs were compared between populations and CNVRs with population differentiated copy numbers were sorted out using the pairwise statistics $V_{S T}$ and Kruskal-Wallis test. Three hundred sixty-two of CNVRs were significantly differentiated in both statistics and 313 genes were located on the population differentiated CNVRs.

Conclusion: For some of these genes, the averages of copy numbers were also different between populations and these may be candidate genes under selection. These include olfactory receptors, pathogen-resistance, parasiteresistance, heat tolerance and productivity related genes. Furthermore, breed- and individual-level comparison was performed using the presence or copy number of the autosomal CNVRs. Our findings were based on identification of CNVs from short Illumina reads of 336 individuals and 39 breeds, which to our knowledge is the largest dataset for this type of analysis and revealed important CNVs that may play a role in cattle adaption to various environments.
\end{abstract}

Keywords: Copy number variation (CNV), Indicine, Taurine, African indigenous cattle, Population differentiated CNV

\section{Background}

Cattle (Bos taurus) has been an invaluable animal providing livestock products such as milk, meat, leather and acting as a draft animal for cultivation and transportation since the domestication of extinct wild aurochs (Bos primigenius) [1]. The two subspecies of Bos taurus, taurine (B. t. taurus) and zebu (B. t. indicus) were brought about after bifurcation in $335,000 \mathrm{BP}$, and were domesticated independently in

\footnotetext{
* Correspondence: heebal@snu.ac.kr

'Interdisciplinary Program in Bioinformatics, Seoul National University, Seoul, Republic of Korea

${ }^{5}$ Department of Agricultural Biotechnology and Research Institute of Agriculture and Life Sciences, Seoul National University, Seoul, Republic of Korea Full list of author information is available at the end of the article
}

different time and location [2, 3]. Archaeological and genomic evidences indicate that the taurine was domesticated approximately 10,000 YBP in Fertile Crescents and the zebu was domesticated 8000 YBP in Indus Valley [4-6]. The domesticated cattle populations were dispersed quickly after domestication along with the migration of pastoralists [5]. Their adaption to various local environments, artificial selection and introgression gave rise to genetically and phenotypically diversified modern cattle breeds [7].

Genome-wide variations such as SNPs and small INDE Ls of cattle were identified in previous studies $[8,9]$. These small variations have been studied for understanding catthe evolution including population structure, selection, 
demographic history and introgression $[7,10,11]$. In case of structural variation, a large proportion in the genome is comprised of CNVs which have great effects on changing of gene structure, dosage and expression level $[12,13]$. In spite of its potentially high functional effects and abundance in the genome, insufficient data and absence of standards in detection and downstream analysis make understanding of CNVs and their impact in cattle genome difficult. However, recent releases of high quality cattle genome assemblies such as ARS-UCD1.2, UOA_Angus_1 and UOA_Brahman_1 make NGS based CNV study available and more credible $[14,15]$. The CNV calling based on short read mapping is now able to detect rare or novel variants, expanded target region to genome-wide, and improved resolution of the location [16].

Here, we detected genome-wide CNVs of 336 individuals in 39 global cattle breeds including Eurasian taurine, Asian indicine and African indigenous cattle, and 2 individuals of African buffalo (Syncerus caffer caffer) using NGS read mapping. This is the largest number of breeds and individuals used in an NGS read mapping based cattle CNV study, including, notably, 19 breeds of African cattle that have not been well understood in terms of their CNVs. CNVs were defined from paired-end mapping result of short reads produced by Illumina HiSeq or NovaSeq platform. We performed population genetics survey on autosomal copy number variation regions (CNVRs). Hierarchical clustering of CNVRs from all individuals were compared to geographical origins and breeds. CNVRs with population differentiated copy number were identified by pairwise comparison of variance and rank based statistics. Population differentiated CNVRs overlapping genes were functionally annotated and suggested as candidate genes associated with selection and adaptation.

\section{Result \\ CNV calling and CNVR definition}

The coverage and sequencing depth of mapped short reads data are important to reliably call CNVs using read depth information. In several previous studies, samples with mean depth coverage over $5 \mathrm{x}$ were used for $\mathrm{CNV}$ analysis, showing that $4 \mathrm{x}$ depth coverage is sufficient for read depth-based CNV detection [17-19]. In our dataset, the minimum mean depth was higher than $5.1 x$, and the mean values of alignment rate, coverage and mean depth of coverage were 99.5, 95.0\%, 11.4x (Table S1). After calling and filtering CNVs, 18,391 CNVRs were identified on autosomes (Table S2), covering 236.2 Mbp or $9.49 \%$ of B. taurus autosomes.

\section{Population differentiation based on CNVR}

In the hierarchical clustering tree based on CNVR, 8 individuals including 1 Maremmana (MAM03), 1 Maronesa
(MAN01), 4 Jersey individuals (JER03, JER04, JER05 and JER06), 1 Angus (ANG09) and 1 Ankole (ANK03) were distant from other individuals (Fig. 1). Except for the 8 individuals, 330 individuals which consisted of 2 AFB, 211 ASI or AFH (indicine group), 117 EAT or AFT (taurine group) were classified by their species and subspecies. Most of the taurine individuals were clustered by their breeds in contrast to indicine individuals. The AFT individuals were clustered by their breeds, and were separated from EAT breeds that were mostly well clustered by their breeds. The four EAT breeds, Holstein, Hanwoo, Hereford and Simmental, were distinguished from other breeds and all individuals in each breed were clustered together. The individuals of two Finn cattle breeds, Western Finn and Eastern Finn, were not distinguished from each other, but clustered together. Six of ten Angus and 9 of 10 Jersey individuals were clustered and differentiated by their breeds. Rest of the taurine individuals included in Maremmana, Podolica, Pajuna, Sayaguesa and Limia from SouthWestern Europe were clustered together. While Nelore and Gir were distinguished from AFH, individuals in other ASI breeds such as Brahman, Sahiwal, Tharparkar and Hariana were clustered with AFH individuals.

The variance of copy numbers of each breed and $V_{S T}$ of every autosomal CNVR were calculated for every breed pairs. The range of $V_{S T}$ is from 0 to 1 , with a higher value indicating a larger difference. The pairwise mean $\mathrm{V}_{\mathrm{ST}}$ of regional population were as following: EAT-AFT, 0.008; EAT-ASI, 0.017; AFH-ASI, 0.023; AFH-EAT, 0.024; AFH-AFT, 0.045; AFT-ASI, 0.128 (Fig. 2). The average of the mean of pairwise $V_{S T}$ in breed level was 0.166. Most of the AFH and ASI were clustered together and N'Dama was clustered with EAT. Muturu was clustered with the 3 Ethiopian humped breeds including Bagaria, Bale and Semien, and separated from others. Several groups of breeds originated from adjacent region including Finn taurine (Eastern Finn and Western Finn), and the Ethiopian zebu (Bagaria, Bale and Semien) were clustered together by their mean $V_{S T}$.

\section{Detection of candidate CNVR differentiated across populations}

In order to detect population differentiated CNVR across 4 groups (AFH, AFT, ASI, and EAT), two statistics were employed. First, pairwise $V_{S T}$ were calculated between all populations except for AFB. Top 1\% and top $0.1 \%$ values were about 0.500 and 0.759 , respectively. The number of CNVRs with the top $0.1 \% V_{S T}$ was 109 in ASI-AFT pair, 2 in ASI-EAT pair and 0 in other pairs. The number of CNVRs with a higher $V_{S T}$ than top $1 \%$ pairs of populations as follows: 1033 in ASI-AFT pair, 31 in EAT-ASI pair, 21 in EAT-AFH pair, 15 in AFHAFT pair and 2 in both ASI-AFH pair and EAT-AFT 

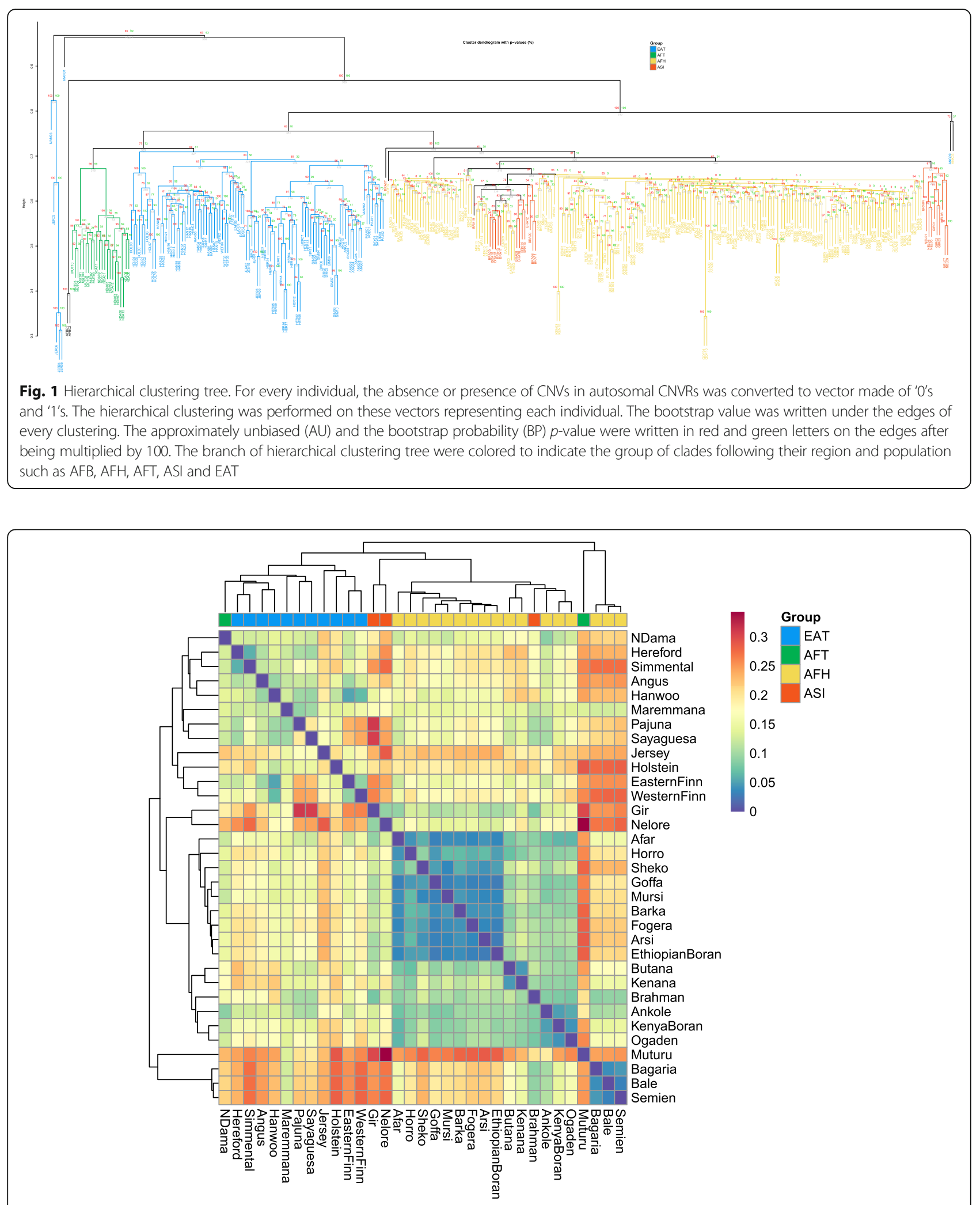

Fig. 2 Manhattan plot of $V_{S T} . V_{S T}$ of CNVRs were visualized as Manhattan plots. The center point of CNVRs was used as $x$-coordinate value. Differentiated genes overlapped with CNVRs significantly different both in upper $1 \% V_{S T}$ and 0.01 significance level of Kruskal-Wallis test on their copy number. The genes whose symbol is starting with 'LOC or differentiated in ASI-AFT pair were left out due to lack of space. The upper $1 \%$ percentile $V_{S T,} 0.500$ and upper $0.1 \%$ percentile, 0.759 were shown as green and red lines respectively 
pair. The $V_{S T}$ of pairs of 4 regional $B$. taurus populations: EAT, ASI, AFT and AFH were visualized as Manhattan plots (Fig. 3). Then, differences in rank of normalized copy number across 4 groups of $B$. taurus including ASI, EAT, AFT and AFH were tested using Kruskal-Wallis test. The population differentiation of CNVRs were determined by the following two criteria: $p$-value under 0.01 in Kruskal-Wallis test and pairwise $V_{S T}$ in upper $1 \%$ which resulted in 910 CNVRs including 313 genes as candidates.

\section{Functional annotation of CNVR overlapping genes}

Among 313 genes overlapped with 362 of population differentiated CNVRs, those with average copy number of four populations including EAT, AFT, AFH, and ASI were summarized in Table S3. The differentiated CNVRs were sorted in ascending order of chi-square from Kruskal-Wallis test. The average copy numbers for AFT, $\mathrm{AFH}, \mathrm{ASI}, \mathrm{EAT}$ groups were written under column for each group. Significantly under- or overrepresented PANTHER GO-slim molecular functions, GO-slim

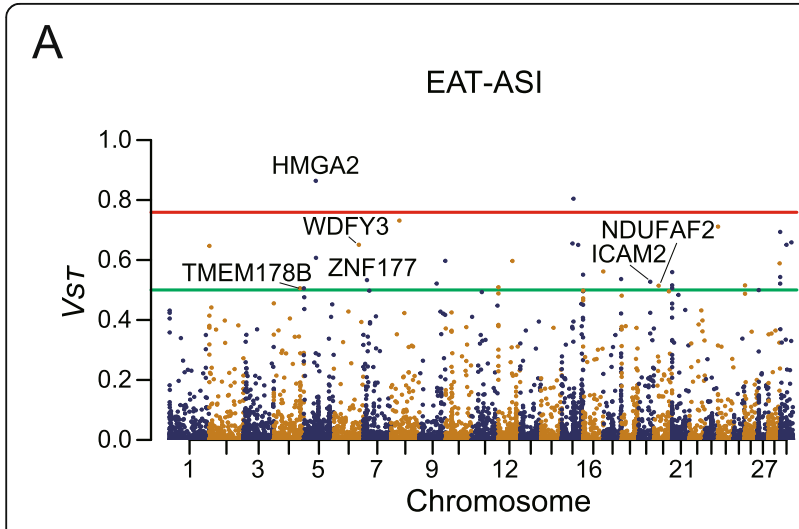

C

EAT-AFH

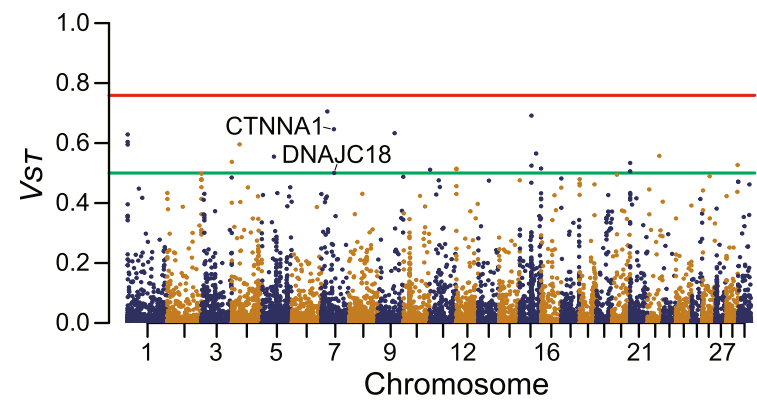

$E$

ASI-AFH

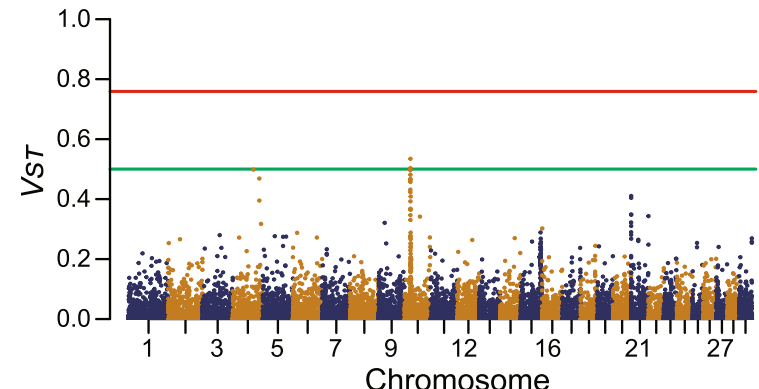

B

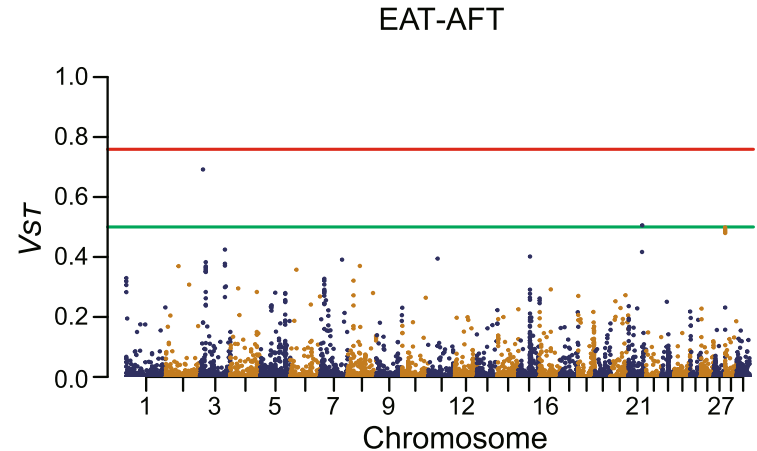

D

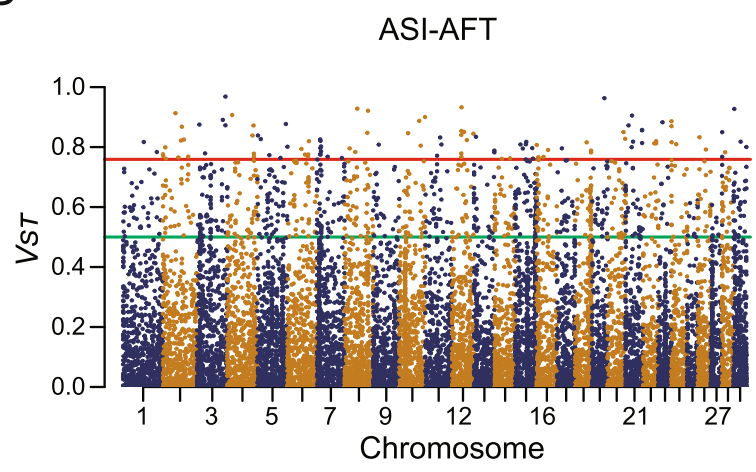

F

AFT-AFH

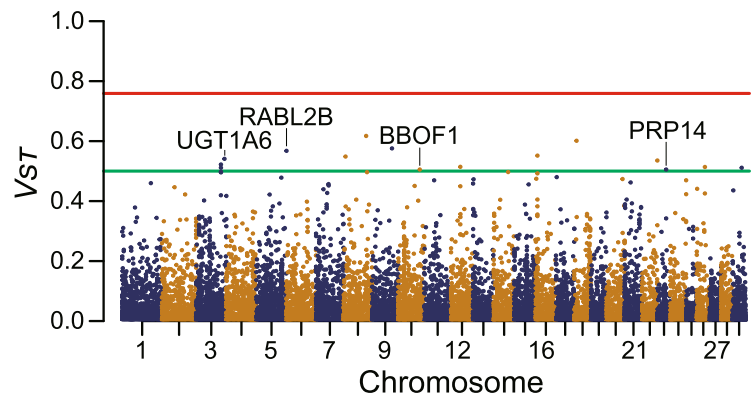

Fig. 3 Mean pairwise $V_{S T}$ values between cattle breeds represented by more than one animal. Clustering tree and heatmap of mean pairwise $V_{S T}$ of autosomal CNVRs. The group of breed was visualized by color above each column. The arrangement of breeds in row and column followed the order by clustering tree. The agglomeration method of clustering was weighted pair group method with arithmetic mean (WPGMA). Breeds were classified to 4 groups by their originated region and taxonomy as follows; AFH, African Humped cattle; AFT, African humpless taurine; ASI, Asian indicus; EAT, Eurasian taurine 
biological processes, or pathways were summarized in Table S4. Most of GO terms with significantly different representation between CNVRs and genome were overrepresented. Regulation associated terms including RNA polymerase II specific DNA binding, DNA-binding transcription factor, regulation of transcription by RNA polymerase II were overrepresented in differentiated CNVRs. Nervous system development and cell differentiation related terms were overrepresented, while immune response and structural constituent of ribosome were underrepresented. Among 72,840 of Autosomal QTLs, 7699 of QTLs overlapped with CNVRs. Five thousand two hundred fifty-two of QTLs overlapped with duplication CNVRs and 2642 of QTLs overlapped with deletion CNVRs. The representation of QTLs related to reproduction, milk and body weight were significantly different compared to total QTL. In reproduction related QTLs, the luteal activity was underrepresented while non-return rate, gestation length and calving ease were overrepresented on CNVRs. Most luteal activity QTL overlapping CNVRs overlapped with duplication while most gestation length QTL overlapped with deletion. The milk content related QTLs such as milk kappa-casein, glycosylated kappa-casein, unglycosylated kappa-casein percentage and milk potassium content were underrepresented on CNVRs. On the other hand, milk fat and yield QTLs were overrepresented. Body weight (yearling) and body weight gain QTLs were underrepresented on CNVRs.

\section{Discussion}

Cattle have been spread with humans across the world after the domestication event in the Fertile Crescent in 10,000 YBP and Indus Valley in 8000 YBP. The genetic diversity of cattle population has been increased by its adaptation to various environments and demographic history including migration and introgression. For example, the population structure of African cattle has diversely changed from its earliest taurine-like population. Since the arrival of $B$. indicus around $700 \mathrm{AD}[20,21]$, the taurine $\times$ indicine cattle admixture event $750-1050$ yr ago [11] and the introgression of African aurochs constructed the complex population structure of the current African cattle. Although population genetics of cattle has been studied extensively based on SNPs, the effects of CNVs on phenotypes and signatures of evolution were poorly understood.

CNVs cover a larger region of genome than SNPs and can impact gene function in multiple ways, including changing of gene structure and dosage, altering gene regulation and exposing recessive alleles [12]. Notably, genes overlapping $\mathrm{CNVs}$ were shown to have better correlations with differentially expressed genes than nearby SNPs, particularly when the CNV overlapped with exons
[22]. Deletions in cattle genome can impact phenotype by interrupting genes and causing loss of biological function [23]. Duplicated genes in cattle genome were related to digestion, lactation, reproduction and immune system such as antigen processing and major histocompatibility genes [13, 24]. CNVs also have population genetic nature related to recombination, mutation, selection, and demography [25]. Generally, CNVs are more recent events than SNPs as they are still segregating within population, showing greater inter-individual variability [16]. These functional impacts and population genetic nature of CNVs have suggested that population differentiation of CNVs may contribute to the phenotypic variation between populations.

Recently, high quality cattle genome assemblies such as ARS-UCD1.2, UOA_Angus_1 and UOA_Brahman_1 increased reliability of $\mathrm{CNV}$ calling and resolution of breakpoint. Above all, Low et al. released haplotyperesolved genome assemblies of of Bos taurus taurus and Bos taurus indicus, and compared CNV between two subspecies [15]. They performed CNV calling using short reads from 38 animals of 7 cattle breeds.

We expanded samples to 336 individuals in 39 global cattle breeds in present study. We aligned short reads on ARS-UCD1.2 assembly to compare larger populations under unified criteria. We identified population stratification of autosome-wide CNVs based on NGS read mapping. Particularly, we included 206 individuals of 19 African cattle breeds in which their genome-wide CNV have been analyzed for the first time in this study.

The traditional classification for African indigenous cattle was based on phenotypes, especially the existence of cervico-thoracic hump. Based on this, some of the hybridized breeds were called Sanga (Zebu $x$ Taurine) and Zenga (Zebu $x$ Sanga). However, genome-wide SNP analysis has identified that the traditional classification did not reflect the genetic difference well [26, 27]. Our CNV based classification generally agreed with previous knowledge with exceptions in several individuals. There were two reasons for the disagreement. First, this study only covered copy number variation region, not the entire genome. Secondly, we compared the read mappingbased copy number, not the sequence itself. Nevertheless, overall concordance of clustering showed potential for population stratification using CNV.

In our CNV-based hierarchical clustering, most individuals were classified by their breeds, whereas some individuals including MAM01, MAM03, ANG09, ANK03 and part of Jersey individuals separated from their breeds. We inspected two possibilities to find the reason for discrepancy. First, we checked similarity between individuals in each breed. We referred to our previous study sharing large part of dataset [11]. The PCA plot and population structure from SNP genotype indirectly 
verified that there were no individuals that significantly distinguished from their breeds. Second, we checked the input vector of hierarchical clustering was the next suspicious factor after excluding the sample problem. It was too simple to represent $\mathrm{CNV}$. The element of vector only considered existence of $\mathrm{CNV}$ on each CNVR, neglecting other properties such as length, breakpoint and copy number of CNV. However, when we tested two other vectors indicating type of $\mathrm{CNV}$ and normalized copy number of $\mathrm{CNV}$, our original vector made a hierarchical tree which was the most concordant with breeds. We speculate that greater inter-individual variability of CNVs compared to SNPs and indels may have contributed to this discordance as well [16].

Mean $V_{S T}$ and the number of CNVRs with high $V_{S T}$ supported the ancestry of African cattle. AFT-EAT and AFH-ASI pairs were relatively similar while the AFTASI pair was mostly different. AFH exhibited high levels of shared CNV with ASI but not with AFT, probably because of the recency of their admixture which was around 150 generations ago [11]. Pairwise comparison of breed distinguished Muturu from other breeds, clustering them with the 3 Ethiopian zebu; Bagaria, Bale and Semien. The African taurine, especially Muturu, showed no evidence of admixture in previous studies assuming EAT and Asian-Australian indicine (AAI) as proxies for unadmixed taurine and indicine cattle, respectively [11]. Muturu was separated from EAT, ASI, and most of AFH except for Bale, Bagaria and Semien in pairwise mean $V_{S T}$ clustering tree. Although the 3 Ethiopian breeds were clustered with Muturu, the mean pairwise $V_{S T}$ did not imply their closeness to Muturu. The mean $V_{S T}$ of Bale, Bagaria and Semien were 0.249, 0.244 and 0.251, respectively, which were all similar with the average 0.249. In addition, Italian taurine, Maremmana (0.132) and the Iberian indigenous taurine, Sayaguesa (0.189) and Pajuna (0.199) have lowest mean $V_{S T} \mathrm{~s}$ against Muturu, which supported the shared ancestry between Muturu and Southern European taurine [11, 28].

Based on the $V_{S T}$ and Kruskal-Wallis test on the copy number of CNVRs, 313 genes were identified as candidate genes under selection and adaptation. Of those, several genes were related to disease susceptibility and resistance. We identified significantly higher copy number of $H M G A 2$ in indicine than in taurine. The indicinespecific copy number gain of HMGA2 was identified by chip-based methods and validated using QPCR in a previous study in which the HMGA2 duplication in Nellore was suggested to be associated with navel length at yearling by haplotype-based GWAS $\left(p=1.01 \times 10^{-9}\right)$ [29] . Navel length at yearling is an economically important trait related to navel injuries in beef cattle. A pendulous navel increases risk of injuries and infection caused by friction against pasture [30]. During natural mating, bulls with long and pendulous navels are frequently exposed to injuries and trauma [31]. Expression of HMGA2 gene is also responsible for body size by regulating myoblast proliferation and myogenesis. $H M G A 2$ directly regulates transcription of $I G F 2 B P 2$ (insulin like growth factor 2 mRNA binding protein 2), and IGF2BP2 promotes myoblast growth. IGF2BP2 regulates translation of IGF1R (insulin like growth factor 1 receptor), $c$ $M y c$, and/or $S p 1$ by binding to their mRNA [32]. Among these genes related to muscle growth, HMGA2, IGF2BP2 and IGF1R overlapped with our CNVRs. The copy numbers of CNVRs overlapping with HMGA2 and IGF1R were significantly different between populations whereas those of IGF2BP2 overlapping CNVRs were not. The copy number of HMGA2 overlapping CNVR was gained in indicine population (EAT: 2.37, AFT: 2.48, AFH: 5.13, ASI: 8.85). On the contrary, the copy number of IGF1R overlapping CNVR was gained in taurine population and lost in indicine population (EAT: 3.28, AFT: 4.34, AFH: 0.92, ASI: 0.43). The knockout mice experiment suggested the positive impact of HMGA2 expression on myoblast growth [32]. On the other hand, Chinese beef cattles with copy number loss of IGF1R had significantly better growth trait such as body weight, body height and hucklebone width [33]. In addition, HMGA2 and IGF1R were strongly associated with size differences between dog breeds [34]. In conclusion, we suggest that differentiated copy number of HMGA2 and IGF1R may be contributing to differences in body size between populations. Copy number variable genes overlapped with taurine-specific duplication such as KRTAP9-1 and KRTAP9-2, and indicine-specific duplication such as CATHL4 and PRDM2 are related to pathogen- and parasite-resistance. The taurine-specific duplication of KRTAP9-1 and KRTAP9-2 corroborates the previous result of comparing copy number of them between European taurine and Asian zebu [18, 35]. They were also identified by aligning WGS short reads to three reference genome assemblies including ARS-UCD1.2, UOA_Angus_1 and UOA_Brahman_1 [15]. The keratin associated proteins were suggested to play a role in tick resistance [36, 37]. Since the cattle skin is the infestation site of tick, the structural protein keratin which makes up the outer layer of skin and hair could act as a barrier [38]. Also, the PRDM2 gene was referred to play a role in resistance to disease and bacterial infection or cellmediated immune response, especially paratuberculosis resistance in ruminants $[39,40]$. The Paratuberculosis (Johne's disease) caused by Mycobacterium avium subspecies paratuberculosis (MAP) brought about considerable economic losses worldwide. The GWAS cohort study about MAP infection in Holstein cattle identified strong signal of SNP and QTL adjacent to PRDM2 gene [41]. Although the resistance to MAP has not yet been 
compared between taurine and indicine, the PRDM2 gene overlapping indicine-specific duplication in our result can be the candidate region for further investigation on adaptation and selection related to paratuberculosis. The higher copy number of CATHL4 in ASI than EUT was also identified in a previous study [18]. The bovine reference genome contains the expanded antimicrobial cathelicidin gene family whereas humans and mice have single copy [42]. Especially, the antimicrobial peptide, indolicidin encoded by CATHL4 can induce autophagic cell death of Leishmana donovani, which is the causative parasite of Leishmaniasis [43]. .The antimicrobial ability which can influence Leishmaniasis lesion development of $C A T H$-family genes was also proved by a knockout in mice [44]. Taken together, the population differentiated $\mathrm{CNV}$ on these genes may contribute to the increased parasite resistance in indicine compared to taurine.

ASI found across the tropical Indian subcontinent adapted to tropical environments characterized with heat stress as well as pervasive pathogen such as tick and parasite [45]. AFH whose ancestry of selection signature skewed toward indicine was also suggested to be adapted to heat stress by indicine introgression into local taurine [11]. In our analyses, one of the heat shock protein family coding gene, DNAJC18 is found to be overlapped with indicine-specific deletion, which is consistent with the CNVR identified in a previous study [46]. The DnaJ family binds to HSP70s for regulating their client capture and drives HSP70s toward specific client [47]. The significantly higher contribution of indicine ancestry [48] and selection signature in East African short horn zebu [49] imply that CNV on DNAJC18 play a role in tropical adaptation and heat tolerance of zebu.

The olfactory function has evolved to alert animals of possible threats such as predators, and provides ability to avoid foods containing parasites, bacteria or chemicals [50]. It also assists animals in locating foods and potential mates [51]. .Olfactory receptors (ORs) play a key role in olfactory function, detecting odor molecules in the olfactory epithelium of the nasal cavity. The OR genes are the largest gene family in the mammalian genome, and there are 881 OR genes in cattle [52]. The OR genes are also characterized by extremely frequent gene duplications and losses [53]. In cattle, about $40 \%$ of OR loci are identified as CNVs. Therefore, the diversity and CNVs on OR genes in cattle could lead to breed specific differences in olfaction capacity [52]. In our result, several OR genes overlapped with the population differentiated CNVRs. There were OR6C202, OR10AD1 and OR5T2 on indicine-specific deletion, OR8U3, OR4C1N, OR4C181, OR2AP1, OR9K2, OR4A16 and OR5D14 on indicine-specific duplication, OR4S1, OR5T2, OR8K1 and OR5AS1 on ASI-specific deletion, OR5M3 and OR5AR1 on ASI-specific duplication and OR8K3,
OR5AS1 and OR5L2 on African cattle specific duplication. As the significant variations in the number and repertoires of OR gene among vertebrates indicate that olfactory function has strongly influenced by natural selection our specific set of OR CNVs might give candidate CNVRs under selection.

Copy numbers of genes associated with quantitative traits related to productivity were frequently gained or lost on cattle genome. In our results, the Eukaryotic translation initiation factor 2 subunit 1 (EIF2S1) gene was overlapped with taurine-specific duplication from 7,927,275.2 to $79,278.2 \mathrm{~kb}$ in chromosome 10 . Copy number on the CNVR in ASI-AFT pair was significant in Kruskal-Wallis test and their $V_{S T}$ was 0.887 . In previous study, EIF2S1 was overlapped with CNVR specific to a high feed efficient group of Holstein [54], which suggests the contribution of the CNVR to different feed efficiency in beef cattle between Bos taurus taurus and Bos taurus indicus [55, 56]. The muscle development related gene CTNNA1 was overlapped with indicine-specific deletion. This result was mostly agreed by $\mathrm{Hu}$ et al. [46] except for the lower copy number in our AFT individuals. The low copy number in Bos taurus indicus while normal or little change in Bos taurus taurus suggest that the sequence is likely to be specific to Bos taurus taurus. The CTNNA1 gene has been described to be associated with myostatin expression level and transcription in skeletal muscle in Holstein-Friesian bulls [57]. Since myostatin plays an essential role in regulating skeletal muscle growth, the taurine-specific existence of CTNNA1 gene would be one of the explanations for difference in meat productivity between Bos taurus taurus and Bos taurus indicus.

\section{Conclusions}

In this study, we explored autosome-wide CNV of global cattle populations and estimated its differentiation between populations. Also, we improved accuracy and resolution of $\mathrm{CNV}$ detection compared to array-based methods and expanded our observation to African indigenous cattle of which $\mathrm{CNV}$ has not been investigated yet. The concordance between population differentiated CNVRs and previous association- and selection-studies supports the possible contributions of CNV to adaptation of cattle. However, our population-scale CNV analyses still have limited accuracy and resolution in detection due to high individual variability and using only one reference genome assembly. When using single reference genome, it would not represent some population enough and it is hard to distinguish CNVs whether minor or major. In further studies, we anticipate that the improvement of reference genome quality and additional high-quality genome assemblies can help solve these problems and enhance the evolutionary interpretation on genome-wide $\mathrm{CNV}$ of cattle. 


\section{Methods}

\section{Sample collection}

The study population consisted of 336 individuals of 39 cattle breeds and 2 individuals of African Buffalo (Syncerus caffer, AFB). Most of individuals except for 10 Bale, 10 Bagaria, 10 Semien and 5 Afar were included in previous SNP-based study by Kim et al. [11]. Names of common individuals here followed the names used in the forementioned study [11]. Breeds of the two subspecies Bos taurus taurus and Bos taurus indicus were collected from Europe, Asia and Africa. Humpless taurine and crossbreeds such as Sanga (Bos taurus taurus $\mathrm{x}$ Bos taurus indicus) and Zenga cattle (Sanga x Bos taurus indicus) were collected from Africa. The 39 Bos taurus breeds were classified into four groups by their original region and subspecies as following: i) 102 individuals of European and Asian taurine (EAT) which included 10 Angus, 10 Holstein, 18 Hereford, 10 Jersey, 11 Simmental, 5 Eastern Finn, 5 Western Finn, 3 Maremmana, 2 Sayaguesa, 2 Pajuna, 1 Limia, 1 Maronesa, 1 Podolica and 23 Hanwoo; ii) 28 individuals of Asian indicine (ASI) which included 16 Brahman, 6 Nelore, 3 Gir, 1 Hariana, 1 Sahiwal and 1 Tharparkar; iii) 22 individuals of African tarurine (AFT) which included 9 Muturu and 13 N'Dama; and iv) 184 individuals of African humped cattle (AFH) which included African zebu and the crossbreeds sanga (zebu $x$ taurine) and zenga (zebu $x$ sanga). The African zebu consisted of 10 Arsi, 10 Bagaria, 10 Bale, 9 Barka, 20 Butana, 10 EthiopianBoran, 10 Goffa, 13 Kenana, 10 KenyaBoran, 10 Mursi, 9 Ogaden and 10 Semien. Sanga consisted of 14 Afar, 10 Ankole and 9 Sheko, and Zenga consisted of 9 Fogera and 11 Horro. Genomes of all individuals were sequenced by Illumina paired-end library and their additional information is described on Table S1. The publicly available sequences were downloaded from SRA with following project accession numbers; PRJNA574857 (Afar, African Buffalo, Arsi, Barka, Butana, Ethiopian Boran, Fogera, Goffa, Horro, Kenana, Mursi, N'Dama, Sheko), PRJNA318087 (Angus, Ankole, Jersey, Kenya, Boran, Kenana, N'Dama, and Ogaden), PRJNA514237 (Limia, Maremmana, Maronesa, Pajuna, Podolica, and Sayaguesa), PRJNA324822 (Brahman), PRJNA343262 (Brahman, Gir, Hereford, Nelore, and Simmental), PRJNA432125 (Brahman), PRJEB28185 (Eastern Finn, and Western Finn), PRJNA210523 (Hanwoo), PRJNA379859 (Hariana, Sahiwal, and Thaparkar), PRJNA210521 (Holstein), PRJNA386202 (Muturu), and PRJNA507259 (Nelore).

\section{Whole genome sequence alignment}

After quality control checking of raw reads using FastQC-0.11.8 [58], adapter and low quality bases of reads were trimmed by Trimmomatic-0.39 [59]. After checking results of trimming and quality of trimmed reads, the trimmed reads were mapped using BWA-
0.7.17 MEM [60] to reference genome ARS-UCD1.2 with Btau5.0.1 Y chromosome assembly. The output of sequence alignment map (SAM) were sorted, indexed and compressed to binary format (BAM) by Samtools1.9 [24]. The duplicates in BAM files were marked using Picard 2.20.2 MarkDuplicates (https://broadinstitute. github.io/picard/) and the marked BAM files were used as input of variant calling. The alignment rate, coverage and mean depth were calculated using Sambamba [61].

\section{CNV calling and CNVR definition}

CNVs of all samples were called with a bin size of 200 bp by CNVnator [62] and filtered with size (>1 kb), $p$ value calculated using t-test statistics $(<0.001)$ and fraction of reads with zero mapping quality $(\mathrm{MQ0}<0.5)$. The CNVs in unplaced scaffolds were removed. A 50\% reciprocal overlap between filtered $\mathrm{CNVs}$ was defined as copy number variation region (CNVR) using 'CNV_overlap.py' script on GitHub (https://github.com/bjtrost/ TCAG-WGS-CNV-workflow) [63]. CNVRs found in more than two individuals were used for downstream analysis to minimize false-positive [64]. Copy number of each CNVR was calculated based on aligned read depth and normalized using CNVnator. The normalized copy number of neutral region from diploid autosome was assumed to be 2.0 .

\section{Hierarchical clustering based on CNVR}

To cluster individuals according to their CNV similarities, we made a vector of " 0 "s and " 1 "s for each individuals based on absence or presence of a specific CNVR in that particular individual. Hierarchical clustering with 1000 bootstrap resampling was performed on these vectors for every autosomal CNVR using pvclust with default option in R [65]. The 'correlation' and 'average' were used as distance measure and the agglomerative method, respectively. The approximately unbiased (AU) $p$-value was calculated by multiscale bootstrap resampling. The bootstrap probability (BP) $p$-value was calculated by ordinary bootstrap resampling based on unweighted pair-group average method (UPGMA).

\section{Population differentiation based on CNVR}

The normalized copy number on CNVRs of all individuals was calculated using CNVnator [62]. $V_{S T}$ of normalized copy number between a pair of breeds, was calculated as $V_{S T}=\left(V_{T}-V_{S}\right) / V_{T}$ where $V_{T}$ is the total variance of normalized copy number among all individuals from both breeds and Vs is the average of variance within each breed, weighted by the number of individuals in the breed [66]. After excluding the 6 breeds with single individual, $V_{S T}$ between pairs of 33 Bos taurus breeds and a buffalo breed were calculated. Mean $V_{S T}$ of all autosomal CNVRs in each pair of breeds were 
visualized using pheatmap in $\mathrm{R}$ [67]. In addition, the $V_{S T}$ of autosomal CNVRs were calculated between EAT, ASI, $\mathrm{AFH}$ and AFT. These results were visualized as Manhattan plots using qqman package in $\mathrm{R}$ [68]. After ranking the normalized copy numbers of all $B$. taurus individuals, Kruskal-Wallist test implemented in 'kruskal.test' $\mathrm{R}$ function were performed on all autosomal CNVRs to compare populations inlcuding EAT, ASI, AFH and AFT . Population differentiated CNVRs were defined as autosomal CNVRs with top $1 \%$ pairwise as well as KruskalWallis test $p$-value less than 0.01 .

\section{Functional annotation of genes overlapped with candidate CNVRs}

Genes overlapped with autosomal CNVRs were annotated based on the reference genome ARS-UCD1.2 from NCBI RefSeq database [69]. In case of genes overlapped with multiple CNVRs, the CNVR with the most significantly different in Kruskal-Wallis test was written. Hypothetical, putative, predicted or uncharacterized genes and pseudo-genes were excluded. The information of functional annotation, gene ontology and pathway of the genes within the population differentiated CNVRs were identified using PANTHER classification system [70]. Comparing the list of genes overlapped with CNVRs with the all genes of Bos taurus in PATHER database [71], we tested the hypothesis whether the PANTHER GO-slim molecular function, GO-slim biological process, and pathway terms were under- or overrepresented in CNVRs using binomial test with Bonferroni corrections [70, 72]. The quantitative trait loci (QTL) underlying CNVRs were also identified using Cattle QTLdb of the reference genome ARS-UCD1.2 [73]. Under- or overrepresentation of autosomal QTL in autosomal CNVRs was tested using binomial test with Bonferroni corrections.

\section{Abbreviations}

CNV: Copy number variation; SNP: Single nucleotide polymorphism; CNVR: Copy number variation region; EAT: Eurasian taurine; ASI: Asian indicus; AFH: African humped cattle; AFT: African taurine

\section{Supplementary Information}

The online version contains supplementary material available at https://doi. org/10.1186/s12864-021-07808-7

Additional file 1: Figure S1. The number of population stratified CNVRs. Venn diagram of the number of population stratified CNVRs.

Additional file 2: Table S1. Sample information and alignment statistics.

Additional file 3: Table S2. Autosomal CNVRs sorted by their genomic region.

Additional file 4: Table S3. Genes overlapped with population differentiated CNVRs. Genes overlapped with significantly different CNVRs based on Kruskal-Wallis test result with $<0.01$ significance level and upper $1 \% V_{\text {ST. }}$. Genes on CNVRs were sorted in ascending order by $p$-values. The pairs of populations with top $1 \%$ or top $0.1 \% V_{S T}$ and the average of copy number of CNVRs in populations including EAT, AFT, AFH and ASI are also indicated.

Additional file 5: Table S4. Over- / underrepresentation of PANTHER GO-slim molecular function, GO-slim biological process and pathway terms on CNVRs.

Additional file 6: Table S5. Over- / underrepresentation of QTLS on CNVRs.

Additional file 7: Table S6. Olfactory receptor genes overlapping population differentiated CNVRs.

\section{Acknowledgements}

The following institutions and their personnel provided help for the sampling of the cattle: The Ethiopian Biodiversity Institute (Ethiopia), The University of Addis Ababa, the cattle keepers from Ethiopia. The ILRI livestock genomics program is supported by the CGIAR Research Program on Livestock (CRP Livestock), which is supported by contributors to the CGIAR Trust Fund (http:// www.cgiar.org/about-us/our-funders/). This research was funded in part by the Bill \& Melinda Gates Foundation and with UK aid from the UK Foreign, Commonwealth and Development Office (Grant Agreement OPP1127286) under the auspices of the Centre for Tropical Livestock Genetics and Health $(C T L G H)$, established jointly by the University of Edinburgh, SRUC (Scotland's Rural College) and the International Livestock Research Institute. The findings and conclusions contained within are those of the authors and do not necessarily reflect positions or policies of the Bill \& Melinda Gates Foundation or the UK Government. The Chinese Government contribution to CAAS-ILRI Joint Laboratory on Livestock and Forage Genetic Resources in Beijing (2018-GJHZ01) is appreciated.

\section{Authors' contributions}

All authors have read and approved the manuscript. JJ and HK conceived and designed the experiments. JJ performed in silico prediction and computational analyses. ET, AT, GB, OH collected samples. HJ generated genome sequencing data. JJ wrote main manuscript text and all of fgures and tables. YHL and KK wrote part of discussion.

\section{Funding}

Not applicable.

\section{Availability of data and materials}

The newly generated sequences for 10 Bale, 10 Bagaria, 10 Semien and 5 Afar individuals are available from Sequence read archive (SRA) with the Bioproject accession number PRJNA698721. (https://www.ncbi.nlm.nih.gov/ sra?linkname=bioproject_sra_all\&from_uid=698721).

\section{Declarations}

Ethics approval and consent to participate

The 35 Blood samples (10 Bale, 10 Bagaria, 10 Semien and 5 Afar individuals) were collected during routine veterinary treatments with the logistical support under the approval of the Ministry of Agriculture, Ethiopia. No further ethics permissions were required for this study. All animals were handled in strict accordance with good animal practice.

Consent for publication

Not applicable.

Competing interests

The authors declared that they have no competing interests.

\section{Author details}

${ }^{1}$ Interdisciplinary Program in Bioinformatics, Seoul National University, Seoul, Republic of Korea. ${ }^{2}$ Addis Ababa University, MCMB Department, Addis Ababa, Ethiopia. ${ }^{3}$ International Livestock Research Institute (ILRI), Addis Ababa, Ethiopia. ${ }^{4}$ Arsi University, Asella, Ethiopia. ${ }^{5}$ Department of Agricultural Biotechnology and Research Institute of Agriculture and Life Sciences, Seoul National University, Seoul, Republic of Korea. ${ }^{6}$ The Centre for Tropical Livestock Genetics and Health (CTLGH), The Roslin Institute, The University of Edinburgh, Edinburgh, UK. ${ }^{7}$ CAAS-ILRI Joint Laboratory on Livestock and Forage Genetic Resources, Institute of Animal Science, Chinese Academy of Agricultural 
Sciences (CAAS), Beijing, China. ${ }^{8}$ Livestock Genetics Program, International Livestock Research Institute (ILRI), Nairobi, Kenya. ${ }^{9}$ School of Life Sciences, University of Notting- ham, Nottingham, UK. ${ }^{10}$ eGnome, Inc., Seoul, South Korea.

Received: 5 March 2021 Accepted: 10 June 2021

Published online: 12 July 2021

\section{References}

1. Magee DA, MacHugh DE, Edwards CJ. Interrogation of modern and ancient genomes reveals the complex domestic history of cattle. Animal Front. 2014:4(3):7-22

2. Achilli A, Bonfiglio S, Olivieri A, Malusa A, Pala M, Kashani BH, et al. The multifaceted origin of taurine cattle reflected by the mitochondrial genome. PLoS One. 2009;4(6):e5753.

3. Loftus RT, MacHugh DE, Bradley DG, Sharp PM, Cunningham P. Evidence for two independent domestications of cattle. Proc Natl Acad Sci. 1994;91(7): 2757-61.

4. Vigne J-D. The origins of animal domestication and husbandry: a major change in the history of humanity and the biosphere. Comptes Rendus Biols. 2011;334(3):171-81

5. Ajmone-Marsan P, Garcia JF, Lenstra JA. On the origin of cattle: how aurochs became cattle and colonized the world. Evol Anthropol. 2010;19(4): $148-57$

6. Chen S, Lin B-Z, Baig M, Mitra B, Lopes RJ, Santos AM, et al. Zebu cattle are an exclusive legacy of the South Asia Neolithic. Mol Biol Evol. 2010;27(1):16.

7. Decker JE, McKay SD, Rolf MM, Kim J, Alcalá AM, Sonstegard TS, et al. Worldwide patterns of ancestry, divergence, and admixture in domesticated cattle. PLoS Genet. 2014;10(3):e1004254.

8. Consortium BH. Genome-wide survey of SNP variation uncovers the genetic structure of cattle breeds. Science. 2009;324(5926):528-32.

9. Hayes BJ, MacLeod IM, Daetwyler HD, Bowman PJ, Chamberlain AJ, Vander Jagt $C$, et al. Genomic prediction from whole genome sequence in livestock: the 1000 bull genomes project. In: Proceedings of the 10th world congress of genetics applied to livestock production: 2014; 2014. p. 17-22.

10. Kim J, Hanotte O, Mwai OA, Dessie T, Bashir S, Diallo B, et al. The genome landscape of indigenous African cattle. Genome Biol. 2017;18(1):1-14.

11. Kim K, Kwon T, Dessie T, Yoo D, Mwai OA, Jang J, et al. The mosaic genome of indigenous African cattle as a unique genetic resource for African pastoralism. Nat Genet. 2020;52:1099.

12. Zhang F, Gu W, Hurles ME, Lupski JR. Copy number variation in human health, disease, and evolution. Annu Rev Genomics Hum Genet. 2009;10: 451-81.

13. Keel BN, Lindholm-Perry AK, Snelling WM. Evolutionary and functional features of copy number variation in the cattle genome. Front Genet. 2016;7:207.

14. Rosen BD, Bickhart DM, Schnabel RD, Koren S, Elsik CG, Tseng E, et al. De novo assembly of the cattle reference genome with single-molecule sequencing. Gigascience. 2020;9(3):giaa021.

15. Low WY, Tearle R, Liu R, Koren S, Rhie A, Bickhart DM, et al. Haplotyperesolved genomes provide insights into structural variation and gene content in Angus and Brahman cattle. Nat Commun. 2020;1 1(1):1-14.

16. Mielczarek M, Fraszzczak M, Nicolazzi E, Williams J, Szyda J. Landscape of copy number variations in Bos taurus: individual-and inter-breed variability. BMC Genomics. 2018;19(1):410.

17. Sudmant PH, Kitzman JO, Antonacci F, Alkan C, Malig M, Tsalenko A, et al. Diversity of human copy number variation and multicopy genes. Science. 2010;330(6004):641-6.

18. Bickhart DM, Hou Y, Schroeder SG, Alkan C, Cardone MF, Matukumalli LK, et al. Copy number variation of individual cattle genomes using nextgeneration sequencing. Genome Res. 2012;22(4):778-90.

19. Consortium GP. An integrated map of genetic variation from 1,092 human genomes. Nature. 2012;491(7422):56.

20. Stock F, Gifford-Gonzalez D. Genetics and African cattle domestication. Afr Archaeol Rev. 2013:30(1):51-72.

21. Hanotte O, Bradley DG, Ochieng JW, Verjee Y, Hill EW, Rege JEO. African pastoralism: genetic imprints of origins and migrations. Science. 2002; 296(5566):336-9.

22. Schlattl A, Anders S, Waszak SM, Huber W, Korbel JO. Relating CNVs to transcriptome data at fine resolution: assessment of the effect of variant size, type, and overlap with functional regions. Genome Res. 2011;21(12): 2004-13.
23. Liu GE, Bickhart DM. Copy number variation in the cattle genome. Function Integrative Genom. 2012;12(4):609-24

24. Liu GE, Ventura M, Cellamare A, Chen L, Cheng Z, Zhu B, et al. Analysis of recent segmental duplications in the bovine genome. BMC Genomics. 2009; 10(1):571.

25. Sjödin $\mathrm{P}$, Jakobsson M. Population genetic nature of copy number variation. Methods Mol Biol. 2012;838:209-23.

26. Bahbahani $\mathrm{H}$, Afana A, Wragg D. Genomic signatures of adaptive introgression and environmental adaptation in the Sheko cattle of southwest Ethiopia. PLoS One. 2018:13:8.

27. Edea Z, Bhuiyan M, Dessie T, Rothschild M, Dadi H, Kim K. Genome-wide genetic diversity, population structure and admixture analysis in African and Asian cattle breeds. Animal. 2015;9(2):218-26.

28. Upadhyay M, Bortoluzzi C, Barbato M, Ajmone-Marsan P, Colli L, Ginja C, et al. Deciphering the patterns of genetic admixture and diversity in southern European cattle using genome-wide SNPs. Evol Appl. 2019;12(5): $951-63$.

29. Aguiar TS, Torrecilha RBP, Milanesi M, Utsunomiya ATH, Trigo BB, Tijjani A, et al. Association of copy number variation at intron 3 of HMGA2 with navel length in Bos indicus. Front Genet. 2018;9:627.

30. Rabelo RE, LAFd S, LAB B, MId M, OCd S, VSd C, et al. Epidemiological aspects of surgical diseases of the genital tract in a population of 12,320 breeding bulls (1982-2007) in the state of Goias, Brazil; 2008.

31. Boligon A, De Vargas L, Silveira D, Roso V, Campos G, Vaz R, et al. Genetic models for breed quality and navel development scores and its associations with growth traits in beef cattle. Trop Anim Health Prod. 2016;48(8):1679-84.

32. Li Z, Gilbert JA, Zhang Y, Zhang M, Qiu Q, Ramanujan $K$, et al. An HMGA2IGF2BP2 axis regulates myoblast proliferation and myogenesis. Dev Cell. 2012;23(6):1176-88.

33. Ma Y-L, Wen Y-F, Cao X-K, Cheng J, Huang Y-Z, Ma Y, et al. Copy number variation (CNV) in the IGF1R gene across four cattle breeds and its association with economic traits. Arch Animal Breed. 2019;62(1):171-9.

34. Sutter NB, Bustamante CD, Chase K, Gray MM, Zhao K, Zhu L, et al. A single IGF1 allele is a major determinant of small size in dogs. Science. 2007; 316(5821):112-5.

35. Bickhart DM, Xu L, Hutchison JL, Cole JB, Null DJ, Schroeder SG, et al. Diversity and population-genetic properties of copy number variations and multicopy genes in cattle. DNA Res. 2016;23(3):253-62.

36. Wang Y, Reverter A, Kemp D, McWilliam S, Ingham A, Davis C, et al. Gene expression profiling of Hereford shorthorn cattle following challenge with Boophilus microplus tick larvae. Aust J Exp Agric. 2007;47(12):1397-407.

37. Nakamura Y, Kanemarum K, Fukami K. Physiological functions of phospholipase C81 and phospholipase C83. Adv Biol Regul. 2013;53(3):356-62.

38. Taye M, Lee W, Caetano-Anolles K, Dessie T, Cho S, Jong Oh S, et al. Exploring the genomes of east African Indicine cattle breeds reveals signature of selection for tropical environmental adaptation traits. Cogent Food Agric. 2018:4(1):1552552.

39. Moioli B, D’Andrea S, De Grossi L, Sezzi E, De Sanctis B, Catillo G, et al. Genomic scan for identifying candidate genes for paratuberculosis resistance in sheep. Anim Prod Sci. 2016;56(7):1046-55.

40. Ghoreishifar SM, Eriksson S, Johansson AM, Khansefid M, MoghaddaszadehAhrabi S, Parna N, et al. Signatures of selection reveal candidate genes involved in economic traits and cold acclimation in five Swedish cattle breeds. Genet Sel Evol. 2020;52(1):1-15.

41. Mallikarjunappa S, Sargolzaei M, Brito LF, Meade KG, Karrow N, Pant S. Uncovering quantitative trait loci associated with resistance to Mycobacterium avium ssp. paratuberculosis infection in Holstein cattle using a high-density single nucleotide polymorphism panel. J Dairy Sci. 2018;101(8):7280-6.

42. Elsik CG, Tellam RL, Worley KC. The genome sequence of taurine cattle: a window to ruminant biology and evolution. Science. 2009;324(5926):522-8.

43. Bera A, Singh S, Nagaraj R, Vaidya T. Induction of autophagic cell death in Leishmania donovani by antimicrobial peptides. Mol Biochem Parasitol. 2003:127(1):23-35.

44. Kulkarni MM, Barbi J, McMaster WR, Gallo RL, Satoskar AR, McGwire BS, Mammalian antimicrobial peptide influences control of cutaneous Leishmania infection. Cell Microbiol. 2011;13(6):913-23.

45. Chan EK, Nagaraj SH, Reverter A. The evolution of tropical adaptation: comparing taurine and zebu cattle. Anim Genet. 2010;41(5):467-77. 
46. Hu Y, Xia H, Li M, Xu C, Ye X, Su R, et al. Comparative analyses of copy number variations between Bos taurus and Bos indicus. BMC Genomics. 2020;21(1):1-11.

47. Kampinga HH, Craig EA. The HSP70 chaperone machinery: J proteins as drivers of functional specificity. Nat Rev Mol Cell Biol. 2010;11(8):579-92.

48. Kasarapu P, Porto-Neto LR, Fortes MR, Lehnert SA, Mudadu MA, Coutinho L, et al. The Bos taurus-Bos indicus balance in fertility and milk related genes. PLoS One. 2017;12(8):e0181930.

49. Bahbahani H, Tijjani A, Mukasa C, Wragg D, Almathen F, Nash O, et al. Signatures of selection for environmental adaptation and zebux taurine hybrid fitness in east African shorthorn zebu. Front Genet. 2017;8:68.

50. Reed DR, Knaapila A. Genetics of taste and smell: poisons and pleasures. Prog Mol Biol Transl Sci. 2010;94:213.

51. Spehr M, Munger SD. Olfactory receptors: G protein-coupled receptors and beyond. J Neurochem. 2009;109(6):1570-83.

52. Lee K, Nguyen DT, Choi M, Cha S-Y, Kim J-H, Dadi H, et al. Analysis of cattle olfactory subgenome: the first detail study on the characteristics of the complete olfactory receptor repertoire of a ruminant. BMC Genomics. 2013; 14(1):596.

53. Niimura Y. Olfactory receptor multigene family in vertebrates: from the viewpoint of evolutionary genomics. Curr Genom. 2012;13(2):103-14.

54. Hou Y, Bickhart DM, Chung H, Hutchison JL, Norman HD, Connor EE, et al. Analysis of copy number variations in Holstein cows identify potential mechanisms contributing to differences in residual feed intake. Function Integrative Genom. 2012;12(4):717-23.

55. Sainz R, Cruz G, Mendes E, Magnabosco C, Farjalla Y, Araujo F, et al. Performance, efficiency and estimated maintenance energy requirements of Bos taurus and Bos indicus cattle. In: Energy and protein metabolism and nutrition in sustainable animal production; 2013. p. 69-70.

56. Canal LB, Fontes PL, Sanford CD, Mercadante VR, DiLorenzo N, Lamb GC, et al. Relationships between feed efficiency and puberty in Bos taurus and Bos indicus-influenced replacement beef heifers. J Anim Sci. 2020;98: skaa319.

57. Sadkowski T, Jank M, Zwierzchowski L, Siadkowska E, Oprządek J, Motyl T. Gene expression profiling in skeletal muscle of Holstein-Friesian bulls with single-nucleotide polymorphism in the myostatin gene $5^{\prime}$-flanking region. J Appl Genet. 2008;49(3):237-50.

58. Andrews S. FastQC: a quality control tool for high throughput sequence data. In: Babraham Bioinformatics, Babraham Institute, Cambridge, United Kingdom; 2010.

59. Bolger AM, Lohse M, Usadel B. Trimmomatic: a flexible trimmer for Illumina sequence data. Bioinformatics. 2014;30(15):2114-20.

60. Li H, Durbin R. Fast and accurate short read alignment with BurrowsWheeler transform. Bioinformatics. 2009;25(14):1754-60.

61. Tarasov A, Vilella AJ, Cuppen E, Nijman IJ, Prins P. Sambamba: fast processing of NGS alignment formats. Bioinformatics. 2015;31(12):2032-4.

62. Abyzov A, Urban AE, Snyder M, Gerstein M. CNVnator: an approach to discover, genotype, and characterize typical and atypical CNVs from family and population genome sequencing. Genome Res. 2011;21(6):974-84.

63. Trost B, Walker S, Wang Z, Thiruvahindrapuram B, MacDonald JR, Sung WW, et al. A comprehensive workflow for read depth-based identification of copy-number variation from whole-genome sequence data. Am J Hum Genet. 2018;102(1):142-55

64. Pierce MD, Dzama K, Muchadeyi FC. Genetic diversity of seven cattle breeds inferred using copy number variations. Front Genet. 2018;9:163.

65. Suzuki R, Shimodaira H. Pvclust: an R package for assessing the uncertainty in hierarchical clustering. Bioinformatics. 2006;22(12):1540-2.

66. Redon R, Ishikawa S, Fitch KR, Feuk L, Perry GH, Andrews TD, et al. Global variation in copy number in the human genome. Nature. 2006;444(7118): 444-54.

67. Kolde R. Pheatmap: pretty heatmaps. R Package Version. 2012;61:617.

68. Turner SD. qqman: an R package for visualizing GWAS results using QQ and manhattan plots. Biorxiv. 2014;1:005165.

69. O'Leary NA, Wright MW, Brister JR, Ciufo S, Haddad D, McVeigh R, et al. Reference sequence (RefSeq) database at NCBI: current status, taxonomic expansion, and functional annotation. Nucleic Acids Res. 2016;44(D1):D733-45.

70. Mi H, Muruganujan A, Ebert D, Huang X, Thomas PD. PANTHER version 14: more genomes, a new PANTHER GO-slim and improvements in enrichment analysis tools. Nucleic Acids Res. 2019;47(D1):D419-26.
71. Mi H, Muruganujan A, Casagrande JT, Thomas PD. Large-scale gene function analysis with the PANTHER classification system. Nat Protoc. 2013; 8(8):1551-66.

72. Nicholas TJ, Cheng Z, Ventura M, Mealey K, Eichler EE, Akey JM. The genomic architecture of segmental duplications and associated copy number variants in dogs. Genome Res. 2009;19(3):491-9.

73. Hu Z-L, Park CA, Reecy JM. Building a livestock genetic and genomic information knowledgebase through integrative developments of animal QTLdb and CorrDB. Nucleic Acids Res. 2019;47(D1):D701-10.

\section{Publisher's Note}

Springer Nature remains neutral with regard to jurisdictional claims in published maps and institutional affiliations.
Ready to submit your research? Choose BMC and benefit from:

- fast, convenient online submission

- thorough peer review by experienced researchers in your field

- rapid publication on acceptance

- support for research data, including large and complex data types

- gold Open Access which fosters wider collaboration and increased citations

- maximum visibility for your research: over $100 \mathrm{M}$ website views per year

At BMC, research is always in progress.

Learn more biomedcentral.com/submissions 